(c) American Dairy Science Association, 2005.

\title{
A Comparison of Three Animal Welfare Assessment Programs on California Dairies
}

\author{
C. L. Stull, ${ }^{1}$ B. A. Reed, ${ }^{2}$ and S. L. Berry ${ }^{3}$ \\ ${ }^{1}$ Veterinary Medicine Cooperative Extension, School of Veterinary Medicine, \\ University of California, Davis 95616 \\ ${ }^{2}$ Glenn County Cooperative Extension, Orland, CA 95963 \\ ${ }^{3}$ Department of Animal Science, University of California, Davis 95616
}

\begin{abstract}
Due to increased awareness of animal welfare issues by consumers, 3 voluntary programs were developed to assess animal welfare on commercial dairies in the United States. These programs include: 1) Humane Farm Animal Care (HFAC), with minimum standards and a third-party audit system; 2) Dairy Quality Assurance Center (DQAC); and 3) University of CaliforniaDavis (UCD) program with self-assessments. The objective of this article was to provide an overview of the similarities and differences in content and scope of these programs. Furthermore, to ascertain the practical application of these programs on commercial dairies, a study was designed to compare the rank order of compliance scores of each program on 10 commercial California dairies using a survey and personal interviews with dairy owners or managers. The survey combined 316 statements from the DQAC checklist, the UCD assessment, and the HFAC standards. The mean ( \pm SD) percentage compliance for the DQAC, HFAC, and UCD programs was $83 \pm 8,94 \pm 3$, and $85 \pm 6 \%$, respectively. A ranking order from 1 to 10 was assigned to each dairy using the compliance score for each assessment program. Comparisons of ranking order between programs were not significant, indicating that different indices were being measured by each program. All programs, however, identified the same 2 lowest-ranking dairies, which indicated that the programs were able to identify facilities with the weakest welfare practices. The survey results show that selection of the available assessment programs for animal welfare on the dairy is important in determining the outcomes.
\end{abstract}

(Key words: animal welfare, auditing, certification, welfare standard)

Abbreviation key: DQAC = Dairy Quality Assurance Center, HFAC = Humane Farm Animal Care, UCD = University of California-Davis.

Received July 14, 2004.

Accepted January 3, 2005.

Corresponding author: Carolyn Stull; e-mail: clstull@ucdavis.edu.

\section{INTRODUCTION}

During the last decade, awareness by consumers of dairy animal welfare issues has increased. Large retail organizations such as the Food Marketing Institute and the National Council of Chain Restaurants have recognized this trend, and responded by requesting animal agriculture to develop and follow guidelines to ensure that animals are handled in a humane manner on the farm (Mench, 2003). To gain consumer confidence, third-party audits were considered essential. Audit materials and protocols for some commodity standards have been established, and auditors currently are being selected and trained to conduct audits at the farm level (Animal Welfare Audit Program, 2003).

In 2002, 2 dairy welfare assessment programs were available for voluntary implementation on commercial dairies. The Dairy Quality Assurance Center (Stratford, IA) revised the 1995 edition of Caring for Dairy Animals-Technical Reference and On-the-Dairy SelfEvaluation Guide (Dairy Quality Assurance Center, 2002). The document contains a self-evaluation guide with a checklist and a technical reference guide with each of 10 quality control points discussed in detail. Administered through the Dairy Quality Assurance Center (DQAC), this program consists of both the written document, and an on-farm audit. The assessors for the on-farm audit are primarily dairy veterinarians and animal scientists trained by the DQAC.

Another nationwide on-farm certification program, Certified Humane Raised and Handled, is based in Virginia, and was initiated in 2002 by Humane Farm Animal Care (HFAC). Humane Farm Animal Care is an independent, nonprofit organization that developed its certification and labeling program through funding resources from the Humane Society of the United States, the American Society for the Prevention of Cruelty to Animals, and regional and local animal welfare organizations. Certified Humane Raised and Handled developed specific minimum standards (HFAC, 2003), certifying protocols, marketing schemes, and a USDA-recognized label for their products in stores. Their standards were based on the Royal Society for the Prevention of 
Cruelty to Animals' Freedom Foods standards in the United Kingdom, but were further developed for US commercial farms by a scientific advisory committee, comprising academic experts from the United States. Third-party assessors visit each dairy to determine compliance with minimum standards before farms are accepted into the program.

Because California is the highest producing dairy state in the United States, producing $20.5 \%$ of the US milk supply (CDFA, 2003), a need exists for the development and implementation of an on-farm, welfare assessment program for dairy facilities in response to rising retail pressure. Commercial dairies in California, however, are different from other dairy locations in the United States due to the geographical and environmental conditions, and a larger average herd size that averaged 776 cows per dairy in 2002 (CDFA, 2003). Thus, the Dairy Welfare Evaluation Guide (Stull et al., 2004) was developed by Cooperative Extension at the University of California-Davis (UCD) for the California Dairy Quality Assurance Program to assess welfare and handling of cattle on commercial dairies. This assessment program is based on the UCD publication written in 1998, Dairy Care Practices (Stull, 1998), along with the publications Recommended Code of Practice for the Care and Handling of Dairy Cattle (Agriculture Canada, 1990), and Caring for Dairy Animals (DQAC, 2002). The module consists of 2 parts: 1) Assessment of the Dairy Facility, which objectively evaluates factors that influence animal welfare on the farm level; and 2 ) the Technical Guide, which provides science-based information on the best management practices for California dairies.

All 3 of the welfare assessment programs cover similar topics such as nutrition, housing, handling, and health. Differences, however, exist in the recommendations and standards among these programs on topics such as the practice of docking tails, transportation of nonambulatory animals to slaughter, opportunity for exercise for all animals, and training of employees on animal welfare issues. Relative differences in compliance scores among the 3 assessment programs may be misleading or confusing to the consumer. The objective of this report is to provide an overview of the similarities and differences in content and scope of 3 voluntary animal welfare assessment programs that are currently available for the commercial dairy. Furthermore, to ascertain the practical application of these programs on commercial dairies, a study was designed to compare the rank order of compliance scores of each program on 10 commercial California dairies using a composite questionnaire and personal interviews with dairy owners or managers.

\section{MATERIALS AND METHODS}

A survey was administered to 10 commercial dairies located in the central valley of California between May and August 2003. Dairies were selected to represent different herd sizes, management styles, breeds of cows, and geographical regions within the valley. None of the dairies was currently enrolled in the HFAC, DQAC, or UCD animal welfare assessment programs. To protect the identity of each dairy, number of cows milked at each facility was categorized into 2 ranges: $\leq 1500$ and $>1500$ cows. The 305-d rolling herd average in kilograms of milk was rounded to the nearest $100 \mathrm{~kg}$ (Table 1). Producers were contacted by telephone to arrange interview times. One or 2 of the authors visited each dairy, read each statement to the dairy representative, and then recorded the answer. Each visit required approximately 90 to $120 \mathrm{~min}$ to complete the survey.

The survey was compiled using 121 statements directly from the Quality Control Points \#1 to 9 Best Management Checklist in the Caring for Dairy Animals (DQAC , 2002), 40 statements from the Assessment of the Dairy Facility of the UCD program (Stull et al., 2004), and 186 statements from the HFAC standards (HFAC, 2003). Statements from the Quality Control Point \#10 in the Caring for Dairy Animals (DQAC, 2002) were omitted from the survey because these statements pertain to biosecurity and environmental stewardship, and not specifically welfare. Because some of the questions on the survey were included in more than 1 program, the survey consisted of 316 statements.

Each statement was read to the dairy representative, and he/she was asked to answer yes, no, or not applicable. A "yes" response denoted that the entire statement supported the current practices and policies of the dairy, whereas a "no" response indicated that the statement was not an accurate description. The "not applicable" response indicated that the statement did not apply to that dairy. Questions were not assigned to major and minor compliance categories, but each made equal contribution to the compliance score. Answers were recorded on the survey sheet and the compliance scores for each dairy calculated.

From the data collected at each dairy, the percentage of "yes" responses divided by the total number of applicable statements in the survey was designated as the percentage compliance score for the individual program. Applicable statements were calculated by subtracting the number of "not applicable" statements from the total number of statements. These results are presented in Table 1, along with the ranking of the dairies from the best to the worst compliance score.

A ranking order from 1 to 10 was assigned to each dairy using the compliance score for the individual as- 
Table 1. Number of cows milked, 305-d rolling herd average (RHA), percentage compliance (\% CPL), and rank designation of dairies ( 1 is best; 10 is worst) for the Dairy Quality Assurance Center (DQAC), Humane Farm Animal Care (HFAC), and the University of California-Davis (UCD) programs.

\begin{tabular}{|c|c|c|c|c|c|c|c|c|}
\hline \multirow[b]{2}{*}{ Dairy } & \multirow{2}{*}{$\begin{array}{l}\text { No. of } \\
\text { cows }\end{array}$} & \multirow{2}{*}{$\begin{array}{l}\text { RHA, } \\
\mathrm{kg}\end{array}$} & \multicolumn{2}{|c|}{ DQAC } & \multicolumn{2}{|c|}{ HFAC } & \multicolumn{2}{|c|}{ UCD } \\
\hline & & & $\% \mathrm{CPL}$ & Rank & $\% \mathrm{CPL}$ & Rank & $\%$ CPL & Rank \\
\hline A & $<1500$ & 8200 & 80.7 & 7 & 95.3 & 4 & 87.5 & 3 \\
\hline B & $<1500$ & 10,900 & 83.9 & 5 & 97.0 & 2 & 82.5 & 8 \\
\hline C & $<1500$ & 10,900 & 85.5 & 3 & 98.2 & 1 & 87.2 & $4 / 5$ \\
\hline D & $<1500$ & 12,300 & 82.8 & 6 & 96.8 & 3 & 87.2 & $4 / 5$ \\
\hline $\mathrm{E}$ & $>1500$ & 9500 & 80.4 & 8 & 93.1 & 7 & 89.5 & 2 \\
\hline $\mathrm{F}$ & $>1500$ & 8200 & 92.1 & 2 & 92.3 & 8 & 84.6 & 7 \\
\hline G & $<1500$ & 7300 & 93.5 & 1 & 93.7 & 6 & 97.5 & 1 \\
\hline $\mathrm{H}$ & $>1500$ & 11,400 & 66.1 & 10 & 88.4 & 10 & 72.5 & 10 \\
\hline I & $>1500$ & 11,400 & 85.2 & 4 & 94.1 & 5 & 85 & 6 \\
\hline J & $>1500$ & 10,400 & 78.3 & 9 & 91.0 & 9 & 80 & 9 \\
\hline
\end{tabular}

sessment program. The Spearman coefficient of rank correlation (Spearman, 1904) was performed on the rank order of the dairies for the 3 programs.

\section{RESULTS AND DISCUSSION}

The 10 dairies represented in the study were located in 4 counties in central California, including Tulare and Merced counties, which are the top milk-producing counties in the state. Average herd size in California (776 lactating cows) is the highest in the nation and the mean $( \pm$ SD) herd size for the study was $1954 \pm$ 2347 cows. Even though the mean herd size of the study dairies was larger than the state average, herd size of 3 of the dairies in the study was 776 cows or less (Table 1 ). The mean 305-d rolling herd average $( \pm$ SD) of the 10 dairies on the study was $9978 \pm 1647 \mathrm{~kg}$ of milk, which is similar to the state average of 9627 , but greater than the national average of $8441 \mathrm{~kg}$ of milk (CDFA, 2003).

Animal welfare is a complex combination of factors that include environment, housing, genetics, health, hygiene, and management. No single factor has been identified that satisfactorily evaluates well-being under all conditions, nor has the contribution of each factor to animal welfare been evaluated or prioritized. Thus, a "benchmark" for dairy animal welfare is difficult to characterize. Selection of assessment criteria that reflects quantitative comparisons is equally challenging. All 3 voluntary assessment programs address guidelines or standards in the following areas: calf management, facilities and environment, handling, health care, management, nutrition, and transportation. In addition, the HFAC program addresses further development of guidelines for slaughter procedures. One major difference among programs is the application of the assessment results. Both the DQAC and the UCD are self-evaluations of a commercial dairy facility, consisting of a useful technical guide to assist producers or other dairy representatives with each of the statements in the self-evaluation. The technical guide provides information for dairy management based on scientific principles or best management practices. A thirdparty auditing system was organized for the DQAC program using trained veterinarians and animal scientists. As of January 2004, approximately 120 auditors have attended the half-day training sessions and been approved by DQAC to perform on-farm audits. It is recommended by DQAC that dairy facilities be selfaudited annually, and by a third-party auditor every 2 yr. Facilities may receive a 5 -star rating when a thirdparty audit score is $80 \%$ or above, and a 4 -star rating when a $70 \%$ audit score is achieved (K. Carlson, personal communication, 2004).

The UCD program provides guidelines for recommended animal welfare practices with no third-party validation. Processors expect their producers-members to evaluate their management and facilities and follow the guidelines to gain consumer confidence. However, the UCD program could be implemented by an independent auditing firm. Both the DQAC and UCD programs quantify the responses to provide a benchmark score, but do not determine a specific passing or failing score. Thus, dairy facilities may be compliant with some, but not all, of the guidelines.

The standards for the HFAC certifying and labeling program were developed based on the philosophical platform of their funding sources including the American Society for the Prevention and Cruelty to Animals and the Humane Society of the United States. These standards are based on the Royal Society for the Prevention and Cruelty to Animals' Freedom Foods program and the Federation of Animal Science Societies' Guide for the Care and Use of Agricultural Animals in Agricultural Research and Teaching (FASS, 1999), but developed for US dairies by an expert scientific committee. Certification of a dairy is granted when the facility is in compliance with all minimum standards. These 
standards are based on the Five Freedoms (Farm Animal Welfare Council, 1983) that include the opportunity for the animals to perform most of their normal behaviors. For example, the HFAC guidelines for calves are specific for compliance in prohibiting tethering or the use of muzzles or physical alterations to prevent inappropriate suckling, but encourage alternative devices such as artificial nipples.

Initially, on-farm HFAC assessment is necessary for certification, with the USDA Agricultural Marketing Service verifying the assessment process. The HFAC program provides the third-party assessor with a checklist of questions corresponding to each of their standards. Each standard is evaluated with a written response of compliance, noncompliance, or not applicable on the checklist by the assessor. Some practices constitute major noncompliance, whereby the producer is rejected from the program. Examples of major noncompliance include using bST, tethering calves, or using electrical prods. Minor noncompliances are defined as those practices that do not compromise the integrity of the animals. Minor noncompliances may be required to be corrected within a specified period. Once accepted into the program, a certified producer can use the label "Certified Humane Raised and Handled," adding to market advantage. All dairies that supply milk to the cooperative or processors must be certified to qualify for the use of the label placed on food products.

The guidelines for many elective procedures that cause pain are very similar among the 3 programs. For example, hot-iron dehorning is the recommended method for routine disbudding procedures in calves between 2 and 10 wk of age in the DQAC and UCD programs, whereas the HFAC allows the procedure in calves up to $4 \mathrm{mo}$ of age. All programs recommend local anesthesia for dehorning older animals.

The programs do not agree on the practice of tail docking. In the HFAC and UCD programs, tail docking is prohibited due to the lack of scientific evidence supporting any benefit. The DQAC does not recommend tail docking, but when performed, it should be done after the heifer is confirmed pregnant. All 3 programs support switch trimming as an alternative practice to tail docking.

The 3 programs address similar topics using the statements in the self-assessments of the DQAC and UCD programs, or the HFAC assessor's checklist. The range in the number of statements for the programs, however, differs approximately 3 -fold, with 40, 121, and 186 queries for the UCD, DQAC, and HFAC programs, respectively. The percentage of "not applicable" responses for the 10 dairies surveyed was 1.3, 6.7, and $12.5 \%$ for the UCD, DQAC, and HFAC assessment programs, respectively. Increase in the percentage of "not applicable" responses for the different programs may reflect the increase in detail and specificity of the statements for the individual program. For example, a statement in the self-assessment for the UCD program states: "Rations meet or exceed the NRC (2001) requirements for growth and production needs." The HFAC has a similar standard, but assigns 3 statements to the topic including: "Cattle fed a wholesome diet which is appropriate to their age and species, fed in sufficient quantity to maintain them in good health, and formulated to satisfy their nutritional needs."

The characteristics for each dairy and the survey results from each program are in Table 1 . Mean percentage $( \pm$ SD) compliance for the DQAC, HFAC, and UCD programs is $83 \pm 8,94 \pm 3$, and $85 \pm 6 \%$, respectively. Percentage compliance was used to rank the dairies from 1 to 10, where 1 was assigned to the dairy with the greatest percentage compliance. Rank order of each dairy based on compliance scores was used to examine when the dairies were relatively similar among welfare assessment programs. Interestingly, only 2 dairies were the same rank in all 3 programs. The dairies $\mathrm{J}$ and $\mathrm{H}$ ranked 9 or 10 , respectively (the worst rank of compliance), in each of the programs. The remaining 8 dairies had a different rank order depending on the program. These 8 dairies and dairy $J$ were within $1 \mathrm{SD}$ of the mean percentage compliance within the individual program, whereas percentage compliance for dairy $\mathrm{H}$ was approximately 2 SD below the mean.

Spearman correlations for the rank order of the dairies based on compliance scores among different programs ranged from 0.37 to 0.43 , which was not different $(P>0.20)$ in any comparison. This weak correlation among the outcomes of these assessment programs indicates that different indices were being measured among programs. In this limited study, however, all assessment programs identified the same 2 lowestranking dairies. This may indicate that the programs were able to identify facilities having common deficiencies and the (relatively) poorest welfare practices.

The statements from each program's survey that received a "no" response on 5 or more dairies are shown in Tables 2, 3, and 4. The statement that is common to all 3 programs is the practice of transporting nonambulatory animals from the dairy. In 2003, nonambulatory animals were usually transported off the dairy directly to slaughter facilities. The Federal Rule (Vol. 69, No. 7; FSIS, 2004) announced on January 12, 2004, however, stated that all nonambulatory disabled cattle presented for slaughter should be condemned. This new rule may drastically curtail the number of nonambulatory cows being transported from the dairy, and increase the number of on-farm euthanasia procedures performed. 
Table 2. Statements from the Dairy Quality Assurance Center (DQAC) self-audit having "no" responses recorded for 5 or more dairies.

\begin{tabular}{ll}
\hline $\begin{array}{l}\text { No. of } \\
\text { dairies }\end{array}$ & Statements in DQAC self-audit \\
\hline 10 & IgG concentrations of all calves are tested within 3 d of birth. \\
8 & $\begin{array}{c}\text { An annual animal care checkup is held to remind employees, management, field staff, or supply companies, etc., of the } \\
\text { importance of animal care, well-being, and comfort. }\end{array}$ \\
8 & Body temperature of calves is measured frequently for the first 2 wk of birth to guide care. \\
7 & A series of written protocols are followed for elective surgeries and procedures that minimize animal discomfort. \\
7 & Written statements relate to animal care. \\
7 & Needles are never reused (more than one animal). \\
7 & Captive bolt equipment and trained employees are available for euthanasia when needed. \\
7 & Calving area is cleaned and disinfected after each calving. \\
6 & Airborne dust is controlled as a way to reduce exposure to microbes. \\
6 & Bedding is raked at each milking (remove soiled sawdust, sand, or other bedding materials), and fresh bedding is added on a \\
6 & regular basis. \\
5 & Scoring of cleanliness or hygiene ratings of animals is done regularly (monthly). \\
5 & Appropriate teat dips (foams, sprays, etc.) are used prior to and after milking. \\
5 & Downers are slaughtered or euthanized on the farm. \\
\hline
\end{tabular}

The HFAC uses minimum standards for compliance, all of which must be met for acceptance into the program and certification. None of the dairies scored total compliance, but the percentage compliance of 4 dairies was greater than $95 \%$, with dairy C scoring $98.2 \%$ (Table 1). However, each of these 4 farms had one or more major noncompliances that would result in rejection from certification status. The major standards of the 4 farms that were recorded as noncompliant included prohibition of treating cows with rbST, feeding antibiotics to boost growth or feed efficiency, and animals leaving the farm that required assistance to walk. The only major noncompliance of dairy $\mathrm{C}$ was the latter. The survey was conducted in May 2003 before the federal regulation concerning the condemnation of nonambulatory cattle presented at slaughter was announced in January 2004. Thus, with the elimination of transporting nonambulatory animals, dairy $\mathrm{C}$ may qualify for certification with the rectification of only 2 minor noncompliance standards.

Common to both the DQAC and UCD's noncompliance lists (Table 2 and 4) are the written protocols for various animal care and health protocols, including euthanasia. Eight of the dairies conducted no annual review of the policies with employees and other staff personnel. Together, the lack of written protocols and

Table 3. Statements from the Humane Farm Animal Care (HFAC) standards with "no" responses recorded for 5 or more dairies.

\begin{tabular}{ll}
\hline $\begin{array}{l}\text { No. of } \\
\text { dairies }\end{array}$ & Questions from HFAC assessor's checklist \\
\hline 5 & $\begin{array}{l}\text { Cattle must not be deliberately fed antibiotics to boost } \\
\text { growth or feed efficiency. } \\
\text { No animal can leave the farm unless it can walk } \\
\text { unassisted. }\end{array}$ \\
\hline
\end{tabular}

policy review indicated that on many dairies, protocols for common procedures such as dehorning, sick animal care, hoof trimming, euthanasia, or teat removal are not written down as formal policy for the individual dairy. Thus, validation of performance of these procedures would be difficult to evaluate. Similar written protocols are submitted as a part of the HFAC application process, and thus, are not shown as noncompliances for the HFAC program. Other prevalent noncompliance items listed for the DQAC program in Table 2 included very specific practices such as testing concentrations of IgG in all calves within $3 \mathrm{~d}$ of birth or controlling airborne dust as a way to reduce microbes.

Differences in assessment scores among programs may be confusing to the consumer who is not familiar with the details of each program. Perhaps a standardized compliance scoring system or a set of specific minimum standards could be developed and applied across all assessment programs to provide the consumer with a basis of comparison.

Some of the limitations of the methodology of this study include the lack of third-party assessments to validate the responses of the dairy representative and the possibility of different interpretations of the questions by the dairy representative. In the authors' opinion, third-party evaluation would be necessary for any animal welfare certification program, especially in ensuring retailer, distributor, and consumer confidence in the program. Producers in our survey may have had varying interpretations of the questions that could have influenced compliance scores. In other words, one producer might interpret the instructions about a "yes" or "no" response very literally, whereas another might interpret a "yes" or "no" response as meaning that they performed or did not perform the action most of the time. Training programs for assessors by the individual 
Table 4. Statements from the University of California-Davis (UCD) assessment with "no" responses recorded for 5 or more dairies.

\begin{tabular}{ll}
$\begin{array}{c}\text { No. of } \\
\text { dairies }\end{array}$ & Statements in UCD assessment \\
\hline 8 & $\begin{array}{c}\text { On-farm policies are reviewed twice annually to remind employees, management, field staff, or other personnel of the } \\
\text { importance of animal care, well-being, and comfort. }\end{array}$ \\
8 & $\begin{array}{l}\text { Written euthanasia protocols exist. } \\
\text { Dairy management and licensed veterinarian(s) cooperatively write health and care protocols that are available for the } \\
\text { following: elective procedures (castration, dehorning, extra teat removal, and hoof trimming). } \\
\text { Dairy management and licensed veterinarian(s) cooperatively write health and care protocols that are available for } \\
\text { the following: sick or injured animal care including nonambulatory animals. }\end{array}$ \\
5 & Nonambulatory animals are not transported off the dairy to market channels or processing facilities. \\
\hline
\end{tabular}

programs may assist in "standardizing" the interpretive evaluations of the assessors. In addition, the questions were not assigned to minor or major compliance categories as in the HFAC program, but each made equal contribution to the compliance score. Because the questions in this survey included standards of all 3 programs, relative difference among programs was likely to be identified by the questionnaire. Furthermore, the rank order of the dairies based on compliance scores of each program was used to examine the similarity among programs, but no significant correlation was found among any of the programs for the rank order indicating different indices were measured by each program. Actual compliance scores for each dairy would differ with implementation of each program due to the differences in their design, objectives, and standards.

\section{CONCLUSIONS}

The results of this survey indicate that selection of the available assessment programs for welfare of animals on the commercial dairies is important to determine outcomes. The 3 programs covered similar topics, but the outcomes reflected each program's design, purpose for assessment, certifying criteria, and differences in specific standards. Comparison of scoring values and ranking order of the dairies among these programs is likely to indicate differences in the standards, not necessarily the welfare differences per se. Use of the assessment results from any of the programs may be beneficial as a guide to improve or define notable deficiencies.

\section{REFERENCES}

Agriculture Canada. 1990. Recommended code of practice for the care and handling of dairy cattle. Communication Branch, Agriculture Canada, Ottawa, Canada.

Animal Welfare Audit Program. 2003. SES, Inc., Lenexa, KS. Online. Available: http://awaudit.org. Accessed July 1, 2004.

CDFA. 2003. California Department of Food and Agriculture, California dairy statistics and trends 2002. Online. Available: http:// dairy.ca.gov/pdf/Annual/2002/stats2002annual.pdf. Accessed July 1, 2004.

Dairy Quality Assurance Center. 2002. Caring for Dairy AnimalsTechnical Reference Guide and On-the-Dairy Self-Evaluation Guide. Agri-Education, Inc., Stratford, IA.

Farm Animal Welfare Council. 1983. Codes of recommendations for the welfare of livestock cattle. Leaflet 701. Min. Agric., Fisheries and Food, MAFF Publications Unit, London, UK.

FASS. 1999. Guide for the Care and Use of Agricultural Animals in Agricultural Research and Teaching. 1st rev. ed. Fed. Anim. Sci. Soc., Savoy, IL.

Food Safety and Inspection Service (FSIS). 2004. 9 CRF 309.2: Antemortem Inspection. USDA FSIS, Washington, DC.

Humane Farm Animal Care. April 2003. Animal care standards. Humane Farm Animal Care, Herndon, VA. Online. Available: http://www.certifiedhumane.com. Accessed July 1, 2004.

Mench, J. A. 2003. Assessing animal welfare at the farm and group level: A United States perspective. Anim. Welf. 12:493-503.

National Research Council. 2001. Pages 258-280 in Nutrient Requirements of Dairy Cattle. 7th rev. ed. Natl. Acad. Sci., Washington, DC.

Spearman, C. 1904. The proof and measurement of association between two things. Am. J. Psychol. 15:72-101.

Stull, C., ed. 1998. Dairy Care Practices. 2nd ed. Univ. of California, Davis. Online. Available: http://www.vetmed.ucdavis.edu/vetext/ INF-DA/INF-DA_CarePrax.pdf. Accessed July 1, 2004.

Stull, C. L., S. L. Berry, B. A. Reed, and M. A. Payne. 2004. Dairy Welfare Evaluation Guide. Cooperative Extension, Univ. of California, Davis. Online. Available: http://www.cdqa.org/ahw/index.htm. Accessed Nov. 15, 2004. 